\title{
Thin Planes of Satellites in $\Lambda$ CDM are not kinematically coherent
}

\author{
Tobias Buck ${ }^{1} \dagger$, Aaron A. Dutton ${ }^{2}$ and Andrea V. Macciò ${ }^{1,2}$ \\ ${ }^{1}$ Max-Planck-Institut für Astronomie, Königstuhl 17, 69117 Heidelberg, Germany \\ email: buck@mpia.de \\ ${ }^{2}$ New York University Abu Dhabi, PO Box 129188, Abu Dhabi, UAE \\ email: dutton@nyu.edu \\ email: maccio@nyu.edu
}

\section{Introduction}

Recently it has been shown by Ibata et al. (2013) that a large fraction of the dwarf satellite galaxies found in the PAndAS survey (McConnachie et al. 2009) and orbiting the Andromeda galaxy are surprisingly aligned in a thin, extended, and kinematically coherent planar structure. The presence of such a structure seems to challenge the current Cold Dark Matter paradigm of structure formation (Ibata et al. 2014, Pawlowski et al. 2014), which predicts a more uniform distribution of satellites around central objects. We show that it is possible to obtain a thin, extended, rotating plane of satellites resembling the one in Andromeda in cosmological collisionless simulations based on this model. Our new 21 high-resolution simulations (see Buck et al. 2015) show a correlation between the formation time of the dark matter halo and the thickness of the plane of satellites. Our simulations have a high incidence of satellite planes as thin, extended, and as rich as the one in Andromeda and with a very coherent kinematic structure when we select early forming haloes. By tracking the formation of the satellites in the plane we show that they have mainly been accreted onto the main object along thin dark matter filaments at high redshift (Dekel et al. 2009, Libeskind et al. 2009, 2011). Our results show that the presence of a thin, extended, rotating plane of satellites is not a challenge for the Cold Dark Matter paradigm, but actually supports one of the predictions of this paradigm related to the presence of filaments of dark matter around galaxies at high redshift.

Using the simulations we analyse in detail the kinematical structure of planes of satellites. Following the observations the kinematical coherence of the planes is characterized by the line-of-sight velocity (Ibata et al. 2013). By doing this we find a high degree of kinematical coherence in our planes. However, investigating the dependence of the number of co-rotating satellites on the viewing angle shows a variance of this number by $30 \%$. These variations are consistent with that obtained from a sample with random velocities. Using instead the clustering of angular momenta vectors of the satellites in the plane results in a better measure of the kinematic coherence. Thus we conclude that the line-of-sight velocity seems to be not a robust measure of the kinematical coherence of the plane. Our planes consist of $\sim 30 \%$ chance aligned satellites. Tracking the satellites in the plane back in time reveals that the planes appear to be a transient feature and not kinematically coherent as would appear at first sight. Thus, from our simulations we predict some of the satellites in the plane of satellites around Andromeda to have a high velocity perpendicular to this plane.

$\dagger$ Member of the International Max-Planck Research School for Astronomy and Cosmic Physics at the University of Heidelberg, IMPRS-HD, Germany. 


\section{References}

Buck, T., Macciò, A. V., \& Dutton, A. A. 2015, ApJ, 809, 49

Dekel, A., Birnboim, Y., Engel, G., Freundlich, J., Goerdt, T., Mumcuoglu, M., Neistein, E., Pichon, C., Teyssier, R., \& Zinger, E. 2009, Nature, 457, 451

Ibata, R. A., Ibata, N. G., Lewis, G. F., Martin, N. F., Conn, A., Elahi, P., Arias, V., \& Fernando, N. 2014, ApJL, 784, L6

Ibata, R. A., Lewis, G. F., Conn, A. R., Irwin, M. J., McConnachie, A. W., Chapman, S. C., Collins, M. L., Fardal, M., Ferguson, A. M. N., Ibata, N. G., Mackey, A. D., Martin, N. F., Navarro, J, Rich, R. M., Valls-Gabaud, D., \& Widrow, L. M. 2013, Nature, 493, 62

Libeskind, N. I., Frenk, C. S., Cole, S., Jenkins, A., \& Helly, J. C. 2009, MNRAS, 399, 550

Libeskind, N. I., Knebe, A., Hoffman, Y., Gottlöber, S., Yepes, G., \& Steinmetz, M. 2011, MNRAS, 411, 1525

McConnachie, A. W., Irwin, M. J., Ibata, R. A., Dubinski, J., Widrow, L. M., Martin, N. F., Côté, P., Dotter, A. L., Navarro, J. F., Ferguson, A. M. N., Puzia, T. H., Lewis, G. F., Babul, A., Barmby, P., Bienaymé, O., Chapman, S. C., Cockcroft, R., Collins, M. L. M., Fardal, M. A., Harris, W. E., Huxor, A., Mackey, A. D., Peñarrubia, J., Rich, R. M., Richer, H. B., Siebert, A., Tanvir, N., Valls-Gabaud, D., \& Venn, K. A. 2009, Nature, 461, 66

Pawlowski, M. S., Famaey, B., Jerjen, H., Merritt, D., Kroupa, P., Dabringhausen, J., Lüghausen, F., Forbes, D. A., Hensler, G., Hammer, F., Puech, M., Fouquet, S., Flores, H., \& Yang, Y. 2014, MNRAS, 442, 2362 